\title{
Diffusive Conductors as Andreev Interferometers
}

\author{
Yuli V. Nazarov and T. H. Stoof \\ Faculteit der Technische Natuurkunde, Technische Universiteit Delft, 2628 CJ Delft, The Netherlands
} (Received 17 August 1995)

\begin{abstract}
We theoretically study phase-dependent electrical transport in diffusive normal metal-superconductor structures, taking into account (a) the effect of electron-electron interaction in the normal metal and (b) the previously known "thermal" effect caused by the energy dependence of the diffusivity. Both effects cause changes in the resistance as a function of the phase between two superconductors, but effect (a) is already present at zero temperature, in contrast to effect (b). A detailed theoretical and numerical analysis demonstrates that the mechanism (b) can fully explain recent experiments by Petrashov et al. [Phys. Rev. Lett. 74, 5268 (1995)].
\end{abstract}

PACS numbers: 74.50.+r, 74.80.Fp

What is the resistance of a small normal structure adjacent to a superconductor? Superconductivity penetrates the structure provided it is short enough. A naive suggestion would be that the resistance vanishes. However, it is not so. The simplest way to see this is to relate the resistivity to the scattering in the structure [1]. Normal electrons traversing the structure should undergo scattering even if their wave functions are distorted by superconductivity.

If the structure is connected to two superconducting terminals having different phases, the resistance of the structure will depend on the phase difference. This provides the physical background for what is called Andreev interferometry. There is an outburst of interest in this topic. Different types of Andreev interferometers have been proposed theoretically [2-4] and realized experimentally [5-8].

Andreev scattering reveals a significant difference between diffusive conductors, from one side, and tunnel or quasiballistic junctions of the same resistance, from another side. Optimal interferometers are composed of tunnel junctions [4,5]. Ballistic and quasiballistic systems also show a big effect [2,7]. In contrast to this, the standard theory predicts that the zero-voltage, zerotemperature resistance of a diffusive conductor is not affected by penetrating superconductivity [9]. It is slightly modified at finite temperature, when the sample length becomes comparable to the superconducting correlation length in the normal metal, $\xi=\sqrt{D / \pi T}, D$ being diffusivity. At higher temperatures, the resistance turns back to the same value. This is why the effect of Andreev scattering on the diffusive resistivity is eventually a thermal effect. Although this fact is well established and has been confirmed in the frameworks of several independent approaches, a simple physical explanation of the fact still is lacking. Apart from this thermal effect, a small modification may arise from the weak localization correction [10].

However, the recent experiment [8] demonstrates a significant phase modulation of the resistivity in the small diffusive structures at very low temperatures. The amplitude of modulation exceeds by several orders of magnitude the one observed in bigger structures at higher temperatures [6]. The authors doubt that their results can be explained by existing theories.

Below we present a novel mechanism that provides zero-temperature phase-dependent resistivity. Because of electron-electron interaction, a weak pair potential is induced in the normal metal that leads to extra Andreev reflection occurring in the structure rather than in the superconductors. The relative change of the resistivity $\delta R / R \simeq g, g$ being the interaction parameter, which may be of either sign depending on the sign of $g$.

However, our numerical results show that for the concrete structure its magnitude is too small to explain the data of Ref. [8]. Careful analysis allowed us to conclude that actually the more trivial thermal effect has been observed. The data show excellent agreement with the results of our simulations.

The most adequate theoretical description of the system is provided in the framework of the Keldysh Green's function technique elaborated in [11] for superconductivity. In the diffusive approximation, one first gets equations for the advanced (retarded) Green's function, which is a $2 \times 2$ matrix, $\hat{G}(x, \epsilon)$, depending on coordinate and energy, with $\hat{G}^{2}=\hat{1}$, whereby

$$
\partial_{x}\left(D \hat{G} \partial_{x}\right)+i[\hat{H}, \hat{G}]=0,
$$

$D$ being the diffusivity in the normal state and $\hat{H}=$ $\epsilon \sigma_{z}+i\left[\operatorname{Re} \Delta(x) \sigma_{y}+\operatorname{Im} \Delta(x) \sigma_{x}\right]$. We will assume that the temperature is low enough and the size of the normal structure is large enough to satisfy $T, D / L^{2} \ll \Delta_{S}$, $\Delta_{S}$ being the energy gap in the superconductors. The boundary conditions for $\hat{G}$ then take a simple form ( $\sigma$ being Pauli matrices): $\hat{G}=\sigma_{z}$ in normal reservoirs and $\hat{G}=\sigma_{x} \sin \phi+\sigma_{y} \cos \phi$ in a superconducting reservoir having phase $\phi$.

The Green's function $\hat{G}$ determines the characteristics of the energy spectrum of the quasiparticles in the structure. To solve the transport problem, we must know how this spectrum is filled by extra quasiparticles. The equation for the even-in-energy part of the filling factor reads

$$
\partial_{x}\left[D(\epsilon, x) \partial_{x} f(\epsilon, x)\right]+\gamma(x) f(\epsilon)=0 .
$$


The first term here describes diffusion of quasiparticles with a diffusion coefficient that is changed by the penetrating superconductivity, $D(\epsilon)=D \operatorname{Tr}\left[\left(\hat{G}+\hat{G}^{+}\right)^{2}\right] / 8$. At zero energy, this corresponds to zero temperature, $\hat{G}=$ $\hat{G}^{+}$and $D$ remains unchanged. The second term describes absorption of quasiparticle excitations into the superconducting condensate, or, in other words, conversion of the normal current into the superconducting one. The coefficient $\gamma$ is proportional to the local value of the pair potential, $\gamma(x)=\Delta(x) \operatorname{Tr}\left(i \sigma_{y}[\hat{G}(\epsilon, x)+\hat{G}(-\epsilon, x)]\right)$.

In a normal reservoir biased at voltage $V$ with respect to superconductors $f(\epsilon)=e V / 4 T \cosh ^{-2}(\epsilon / 2 T)$. This provides boundary conditions for (2). The current into a reservoir is determined by the local gradient of $f$.

The common theoretical approach (see, for instance, $[1,4,12])$ disregards interactions in the normal metal that leads to $\Delta, \gamma \equiv 0$. Since for diffusive conductors at zero temperature the common noninteracting picture does not give the resistance change, we concentrate on the effect of $\Delta$ in the normal metal. This value can be calculated with

$$
\Delta=g \int d \epsilon \tanh (\epsilon / 2 T) \operatorname{Tr}\left[i \sigma_{y}\left(\hat{G}^{A}-\hat{G}^{R}\right)\right] / 8 .
$$

This is the novel feature of the present approach.

Let us first make a simple estimation of the magnitude of the effect. Since it is expected to be small, we solve Eq. (2) to first order in $\gamma$. This gives a relative resistance change $\delta R / R \simeq \gamma L^{2} / D$. At zero energy, $\gamma \simeq \Delta$. In normal metal the energies in the window $\simeq D / L^{2}$ contribute to $\Delta$, therefore $\Delta \simeq g D / L^{2}$. This results in a simple estimation for the resistance change,

$$
\delta R / R=g c(\Phi),
$$

$c$ being a dimensionless number depending on the geometry of the structure and on the distribution of the resistivity therein. It is important to note that $c$ depends neither on the structure size nor on the absolute value of the resistivity, provided the temperature is low enough, $T \ll D / L^{2}$. The effect depends on the normal metal material by means of $g$ and can be of either sign depending on the sign of $g$. If the geometry of the structure is well defined, the effect can be used for the direct measurement of interaction in normal metal.

At a qualitative level, the effect seems to explain the results of Ref. [8]. Indeed, the phase modulation of the resistance they observed at low temperatures was of the order of several percent and appeared material dependent, including the sign of the resistance change. This prompted us to make a detailed numerical calculation of the resistance of a concrete structure (Fig. 1) which is very similar to the one used in Ref. [8].

The structure consists of the current branch, the superconducting branch connected to superconducting reservoirs biased at the phases $-\Phi / 2, \Phi / 2$, respectively, and the extra branch made for technological reasons. The current flows as it is shown in Fig. 2 and the voltage difference between the points $A$ and $A^{\prime}$ is measured. Owing

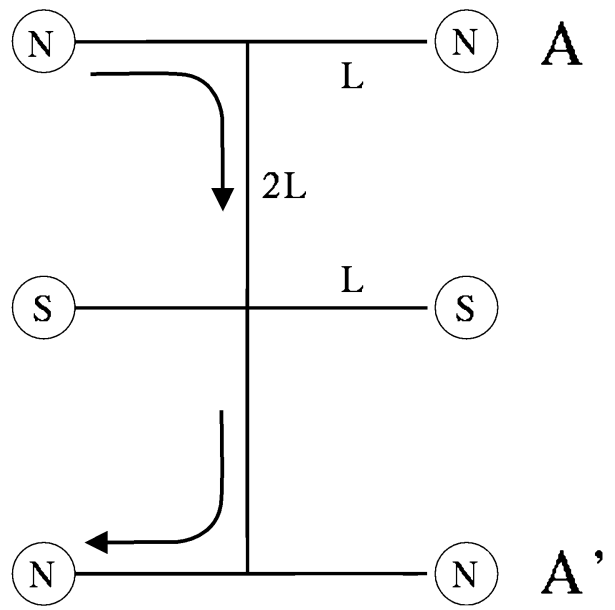

FIG. 1. The structure under consideration.

to the symmetry of the structure, the voltage and $f$ distributions are antisymmetric with respect to the superconducting branch, whereas $\Delta(x)$ and $\hat{G}(x)$ are symmetric. Superconductivity in the structure gets completely suppressed when $\Phi$ approaches $\pi$.

One-dimensional differential equations (1) and (2) shall be solved for each branch and then matched in the crossing points. First we calculate $\hat{G}(\epsilon)$ in all points of the structure. Because of the boundary conditions, it depends on $\Phi$ in each point, thus providing the origin of the phase dependent effect. We obtain $\Delta(x)$ by integrating $\hat{G}$ over energy. Then we calculate $\gamma(x)$ and make use of an analytical formula that relates the resistance change to $\gamma(x)$. Details of the calculations will be reported elsewhere [13].

In Fig. 2 we have shown the calculated phase dependence normalized by its maximal value at $\delta \phi=0$, $c(\phi) / c(0)$, contrasted with the experimental data for $\mathrm{Ag}$. The phase dependencies look similar, but the magnitude of the effect cannot be satisfactorily explained. According to the calculation, $c(0)=0.14$. If we take an expected value $g=0.04$ [14] for silver, we would

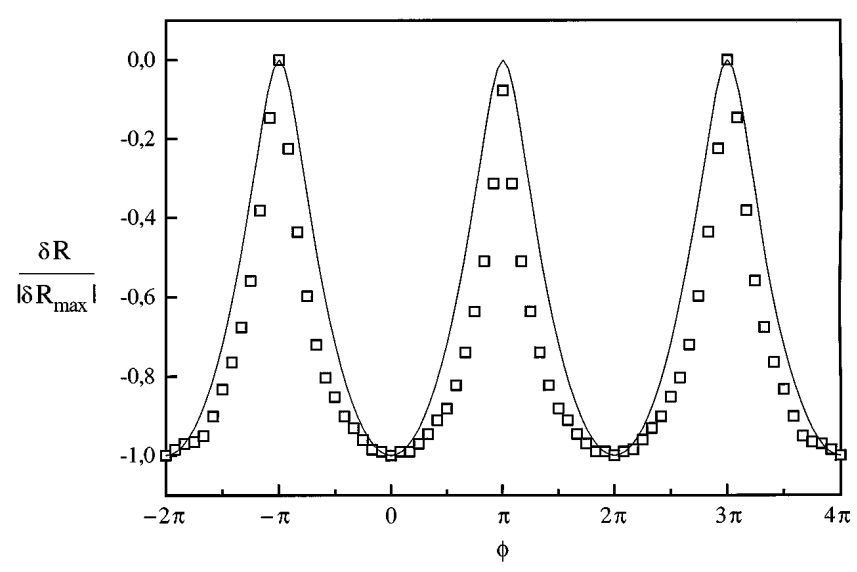

FIG. 2. Normalized phase dependence of the novel effect. Squares correspond to the experimental data of Ref. [6]. 
obtain $\delta R_{\max } / R=0.003$, whereas the experiment gives $\delta R_{\max } / R=0.1$. If we do it the other way around and try to fit $g$ from the experiment, we end up with $g \simeq 0.7$. That would bring silver to the rank of high temperature superconductors.

This prompted us to check the possibility that seemed to be rejected by the authors of Ref. [8]. We have calculated the effect of Andreev reflection on the resistivity neglecting interaction corrections. This arises from the energydependent change of the diffusion coefficient in Eq. (2). The calculation goes as follows. First, we compute using Eq. (1) and the definition of the renormalized diffusion coefficient the actual energy-dependent diffusion coefficient throughout the structure. As we mentioned above, the zero-energy diffusion coefficient coincides with the one at high energy. Energy dependence of diffusion coefficient in a point separated by the distance $d$ from the nearest superconductor has a maximum at energy $\simeq D / d^{2}$. The maximum value exceeds zero or high energy value by $\simeq D$. Owing to boundary conditions for Eq. (1), the diffusion coefficient also depends on the phase of the superconductors. Second, we solve Eq. (2) neglecting $\gamma$. The temperature enters (see above) the boundary condition for this equation and determines the energy interval in which the energy and the phase dependence of the diffusion coefficient are actually felt by the propagating quasiparticles. Therefore we expect the effect to level off at both low and high temperatures giving minimal resistance at temperatures $\simeq D / L^{2}$. In general, for a given geometry, the relative resistance change $\delta R / R$ is a function of $L / \xi$ and $\phi$.

Our numerical results are presented in Fig. 3. As expected, the effect vanishes at both low and high temperatures. The resistance at $\Phi=0$ reaches the minimum at $L \approx 3 \xi$. We have plotted the normalized phase dependence at $L=3 \xi$ in Fig. 4 along with experimental data

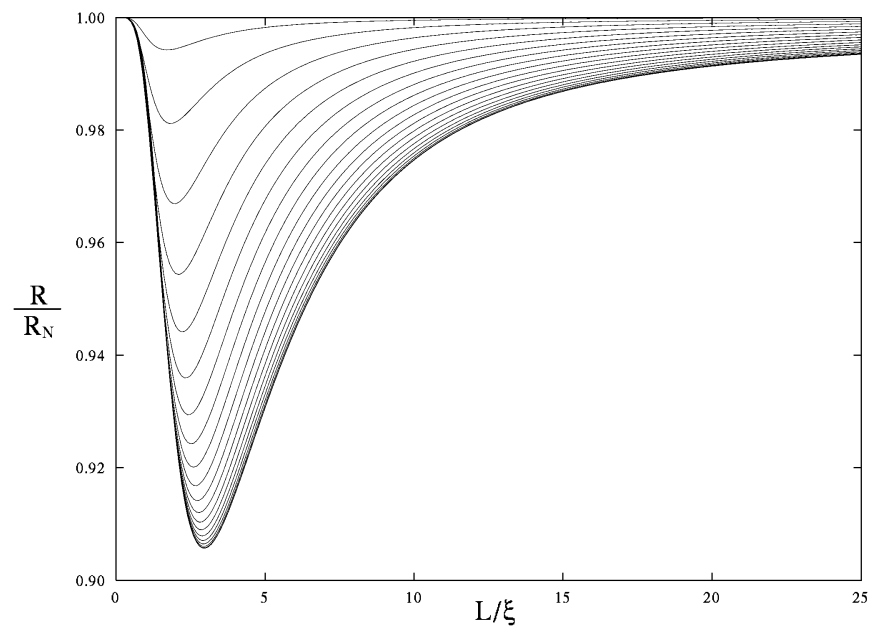

FIG. 3. The temperature and the phase dependence of the thermal effect. The temperature is incorporated into $\xi^{2}=$ $D / \pi T$. The phase difference changes from 0 for the lowermost curve to $\pi$ for the uppermost one with step $\pi / 20$. and obtained a perfect match. The maximal values of the change are also very close to each other: $\left(\delta R_{\max } / R\right)_{\text {theor }}=$ -0.097 vs $\left(\delta R_{\max } / R\right)_{\exp }=-0.11$. From the estimations given in [8] we obtain, indeed, $L / \xi=2.5-3$ at $T=$ $20 \mathrm{mK}$. As we can see in Fig. 3, the thermal effect persists at rather high temperature, in agreement with the long high temperature tail observed in [8]. Unpublished data of the authors of [8] show an excellent agreement with the curves plotted in Fig. 3 [15].

All this allows us to conclude that the experiment [8] can be perfectly described by existing semiclassical theory of superconducting proximity effect and thus to remedy the seeming discrepancy between theory and experiment.

The remaining discrepancy for metallic samples can be easily understood if one takes into account the sensitivity of the effect to a concrete geometry and to the inevitable inhomogeneity of these ultrasmall structures. This point of view is supported by large sample-to-sample fluctuations of the magnitude of the effect [8]. For instance, the understanding of the results for Sb samples having high resistivity presents a certain difficulty. However, we notice that all essential features for these samples, such as small magnitude of the effect, sinusoidal phase dependence, positive sign, and the long high temperature tail, can be well understood if the structures are not completely diffusive but contain tunnel junctions [4].

We are ready to present several conclusions.

We have shown that the results of [8] can be perfectly explained within the existing theoretical framework and be attributed to the thermal effect, provided a concrete experimental geometry is taken into account.

We present a novel mechanism of phase-dependent resistance in hybrid normal metal-superconductor structures that works at zero temperature. The observation of this effect would allow a direct measurement of the interaction parameter in a normal metal.

Our results show that the observation of the weak localization correction [10] is a more difficult task than

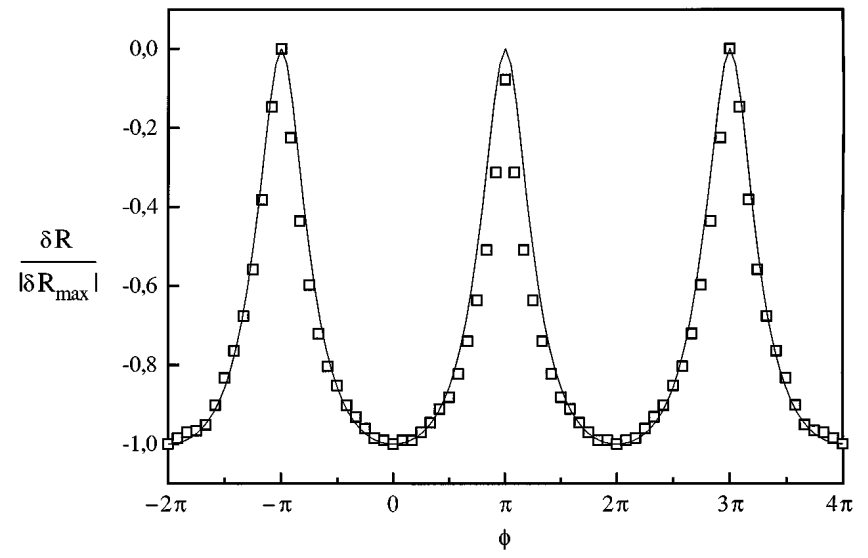

FIG. 4. Normalized phase dependence of the thermal effect at $L=3 \xi$. Squares correspond to the experimental data of Ref. [6]. 
it was thought to be. At zero temperature, this correction will be masked by the novel effect we discussed, provided the interaction is not very small. At high temperatures, the correction would become comparable with the tail of the thermal effect at $T>D R_{Q} / L^{2} R$. For the structures used in Ref. [8] this would correspond to unreasonably high temperatures of $20 \mathrm{~K}$.

The authors are indebted to V. Petrashov for numerous discussions of his results, B.Z. Spivak for his illuminating remarks concerning the interaction in normal metal, D. Esteve, M. Devoret, H. Pothier, and S. Gueron for the discussion from which this work has emerged, A.V. Zaitsev and G.E.W. Bauer for helpful comments, and P. Delsing for the critical reading of the manuscript and communications concerning his unpublished data. This work is a part of the research program of the "Stichting voor Fundamenteel Onderzoek der Materie" (FOM), and we acknowledge the financial support from the "Nederlandse Organisatie voor Wetenschappelijk Onderzoek" (NWO).

[1] C. J. Lambert, J. Phys. Condens. Matter 3, 6579 (1991); C. W. J. Beenakker, Phys. Rev. B 46, 12841 (1992).

[2] H. Nakano and H. Takayanagi, Solid State Commun. 80, 997 (1991); C. W. J. Beenakker, J. Melsen, and P. Brouwer, Phys. Rev. B 51, 13883 (1995); A. Kadigrobov,
A. Zagoskin, R. I. Shekhter, and M. Jonson (to be published); N. K. Allsopp, J. Sanchez Canizares, R. Raimondi, and C. J. Lambert (to be published).

[3] F. W. J. Hekking and Yu. V. Nazarov, Phys. Rev. Lett. 71, 1625 (1993); A. V. Zaitsev, Phys. Lett. A 194, 315 (1994).

[4] Yu. V. Nazarov, Phys. Rev. Lett. 73, 1420 (1994).

[5] H. Pothier, S. Gueron, and D. Esteve, Phys. Rev. Lett. 73, 2488 (1994).

[6] P. G. N. de Vegvar, T. A. Fulton, W.H. Mallison, and R. E. Miller, Phys. Rev. Lett. 73, 1416 (1994).

[7] A. Dimoulas, J. P. Heida, B. J. van Wees, T. M. Klapwijk, W.v.d. Graaf, and G. Borghs, Phys. Rev. Lett. 74, 602 (1995).

[8] V.T. Petrashov, V. N. Antonov, P. Delsing, and T. Claeson, Phys. Rev. Lett. 74, 5268 (1995).

[9] S. N. Artemenko, A. F. Volkov, and A. V. Zaitsev, Solid State Commun. 30, 771 (1979); see also Refs. [1,4].

[10] B.Z. Spivak and D.E. Khmelnitskii, Pis'ma Zh. Eksp. Teor. Fiz. 35, 334 (1982) [JETP Lett. 35, 412 (1982)].

[11] A. I. Larkin and Yu. V. Ovchinninkov, Zh. Eksp. Teor. Fiz. 68, 1915 (1975) [Sov. Phys. JETP 41, 960 (1975)]; A. I. Larkin and Yu. V. Ovchinninkov, Zh. Eksp. Teor. Fiz. 73, 299 (1977) [Sov. Phys. JETP 46, 155 (1977)].

[12] A. F. Volkov, A. V. Zaitsev, and T. M. Klapwijk, Physica (Amsterdam) 210C, 21 (1993).

[13] T.H. Stoof and Yu. V. Nazarov (to be published).

[14] A. C. Mota, P. Visani, and A. Pollini, J. Low Temp. Phys. 76, 465 (1989).

[15] P. Delsing (private communication). 